\title{
Erratum
}

\section{Erratum: Boyer et al., "Mammalian TRIM67 Functions in Brain Development and Behavior"}

In the article "Mammalian TRIM67 Functions in Brain Development and Behavior", by Nicholas P. Boyer, Caroline Monkiewicz, Shalini Menon, Sheryl S. Moy, and Stephanie L. Gupton, which published online May 29, 2019, the mouse inbred strain was listed incorrectly in the Material and Methods section. In the first sentence, "C57BL/7" should instead be "C57BI/6J".This error does not affect the conclusions of the article. The article has been corrected online.

https://doi.org/10.1523/ENEURO.0281-19.2019 